\title{
Lehrbuch Prävention und Gesundheitsförderung
}

\author{
K. Hurrelmann, T. Klotz, J. Haisch \\ Bern: H. Huber (2004)
}

Das vorliegende Werk ist das erste Lehrbuch für Prävention und Gesundheitsförderung, das im deutschsprachigen Raum entstanden und von deutschsprachigen Experten verfasst worden ist.

Man kann es als Meilenstein bezeichnen, dass nun ein solches Buch vorliegt. In dem Sammelband dominiert zwar der präventive Blick, aber in verschiedenen Einzelbeiträgen ist es sehr gut gelungen, den neueren Ansatz der Gesundheitsförderung eigenständig darzustellen oder komplementär zur Prävention miteinzubeziehen.

Die Herausgeber haben sich viel vorgenommen, vor allem, was die Vielfalt der Themen und das breite Spektrum von Methodik und Profession angeht. Dass in diesem Zusammenhang manchmal Unschärfen entstehen zwischen Prävention und Gesundheitsförderung erstaunt nicht.

Den Herausgebern und Herausgeberinnen ist es jedoch gelungen, ein anspruchsvolles, komplexes Unterfangen zu realisieren. Gelungen ist, dass sie für die jeweiligen Themen ganz gezielt ExpertInnen eingeladen haben: GesundheitswissenschafterInnen, SozialwissenschafterInnen, MedizinerInnen und ÖkonomInnen. Das hat den Nachteil, dass zwar Redundanzen entstanden sind, aber die einzelnen Artikel in sich dafür sehr fundiert sind und jeweils eine gute Grundlage für das jeweilige Thema darstellen.

Angenehm und hilfreich ist die knappe Leseempfehlung nach jedem Artikel. Zusätzlich steht eine ausführliche Literaturliste mit ausgewählter, meist deutschsprachiger Literatur zur Verfügung.
Das Buch umfasst sechs Kapitel. Es beginnt mit den Grundlagen und Konzepten von Prävention und Gesundheitsförderung. Dann folgen Artikel zu den unterschiedlichen Lebensalter. Je ein weiteres Kapitel befasst sich mit der Prävention epidemiologisch relevanter Krankheiten, mit fächerspezifischer Prävention und mit dem Zielgruppen- und Settingansatz. Abgeschlossen wird das Werk mit dem Thema gesundheitspolitische Umsetzung.

Etwas schade erscheint, dass Artikel zur konkreten Umsetzungsthematik fehlen. Dies betrifft insbesondere Konzepte und Instrumente zu Planung, Umsetzung und Evaluation und der Qualitätsentwicklung auf Projektebene. Auf diesen Gebieten wurden in den letzten Jahren substantielle Grundlagen entwickelt. Und - sie sind für die wirkungsvolle Umsetzung von Projekten unentbehrlich. Solche Themen hätten sehr gut zum Buch gepasst und die umfassende Thematik abgerundet, zumal Prävention und Gesundheitsförderung keine theoretische Disziplin, sondern zwei Handlungsfelder darstellen.

Trotz diesen Mängeln ist es ein wichtiges und gutes Grundlagenwerk, das in jedes Büchergestell von PraktikerInnen aus ganz unterschiedlichen Disziplinen - sei es inner- und ausserhalb der Gesundheitsprofession - gehört und ein Stück zur Professionalisierung in den Bereichen Prävention und Gesundheitsförderung beiträgt.

Brigitte Ruckstuhl Bern e-mail: brigitte.ruckstuh!@promotionsante.ch 